\title{
The Mechanism and Strategy of Blockchain Technology Driving the Development of Supply Chain Finance
}

\author{
Songqin $\mathrm{Ye}^{1}$, Jiangjiarui Zeng ${ }^{2, *}$ \\ ${ }^{1,2}$ School of Finance, Jiangxi Normal University, Nanchang 330022, China \\ *Corresponding author. Email: jiarui_zengjiang @ 163.com
}

\begin{abstract}
This article is based on the security, traceability, immutability and transparency of blockchain technology, aiming at the pain points of information asymmetry, financing difficulties, expensive financing, and weak supervision in the development of supply chain finance, to study how to use the core technologies of distributed ledgers, peer-to-peer transmission, associated credit, and smart contracts in blockchain technology to empower supply chain finance, expand the scope of blockchain technology, and provide technical support for the supply chain financial model. Strategic cooperation system to conduct innovative research on the combination of supply chain finance and blockchain technology.
\end{abstract}

Keywords: Supply chain finance, Blockchain technology, Information asymmetry, Distributed ledger, Intelligent contract.

\section{INTRODUCTION}

The industrial process of mankind has entered the power industry from the age of steam, and then entered the information society through the scientific and technological revolution. With the rapid development of big data, Internet technology and block chain technology, digital technology has profoundly influenced and reshaped all walks of life, and supply chain finance has been increasingly closely combined with digital technology. Scientific and technological innovation promotes the reform and development of supply chain finance, deepens the technological degree of financial products and services, and provides new ideas for its development and innovation. The Internet and big data have made supply chain financial services informationalized and knowledge-based, blurred the boundaries of financial service institutions, greatly promoted the development of supply chain finance, and optimized resource allocation and capital circulation channels. As a fusion technology of point-to-point communication, digital encryption, distributed ledger, multi-party collaborative consensus algorithm and other fields, blockchain technology has been widely recognized in China's economic field, especially in the financial industry, with the characteristics of transparent and untamper-able information and traceable data on the chain. However, a series of problems in supply chain finance, such as information barrier, expensive financing and difficult supervision caused by information asymmetry, need to be solved urgently. Through block chain technology, can ensure the data confidence, mutual recognition of circulation, the core enterprise credit, to prevent performance risks, improve the efficiency of the coordination, reduce business costs, effectively solve the traditional supply chain, there exists a series of financial problems, the supply chain financial fu, encourage innovation in the supply chain to realize the new development of new financial forms.

\section{THE THEORETICAL BACKGROUND ANALYSIS OF BLOCKCHAIN TECHNOLOGY TO PROMOTE THE DEVELOPMENT OF SUPPLY CHAIN FINANCE}

The earliest appearance of the term blockchain was a concept mentioned by a researcher with the pseudonym "Satoshi Nakamoto" in an article published in 2008 [1]. The article mentioned a concept that was completely passed through P2P (Peer- To-Peer) technology realizes the network transaction system, in this mode, any node of each user can be used as an information storage 
center, so as to achieve the decentralization purpose through the decentralization of information storage and computing, and complete this system The core technology of the company is the blockchain. Although the concept of blockchain aroused heated discussions at the time, a comparative study by $\mathrm{Xu}$ Zhenyu, Wu Jinping, and Huo Yurong found that there are few domestic studies related to it, and they are basically in a stagnant state [2]. However, with the application of Bitcoin, the academic community has gradually realized that blockchain is a universal technology and solution with the advantages of security, immutability and transparency [3]. Ouyang Liwei et al. also pointed out that with the derivative development of blockchain technology and the extension of its application scenarios, blockchain technology has penetrated into many fields such as finance, medical care, and the Internet of Everything. The traditional supply chain finance seems to be linked in the same industrial network, but the actual information exchange frequency is low, difficult, resource exchange efficiency is low, and users at all levels lack trust, leading to greater financial risks [4].

The blockchain is essentially a decentralized distributed system. The information of each node can be operated and calculated independently according to the rules. The entire system is maintained by all participants. The privacy is protected by the key, and the public key is also available at the same time. To build a common information system, every step of the operation is recorded, and at the same time, the entire system will not be broken due to a node's error. Blockchain technology is due to its decentralized point-to-point model and non-tamperable characteristics. Makes information storage and transmission more secure, and work efficiency is maximized. Therefore, this makes it very suitable for supply chain finance scenarios, which will greatly alleviate the principal-agent problem caused by information asymmetry in the supply chain finance industry [5]. To meet the data security and credibility issues that enterprises are paying more and more attention to, and optimize the allocation of resources.

\section{ANALYSIS OF THE GOALS AND DEMANDS OF BLOCKCHAIN TECHNOLOGY TO PROMOTE THE DEVELOPMENT OF SUPPLY CHAIN FINANCE}

In the context of the post-Internet era revolution, the integration of supply chain finance and Internet technology has become more and more closely, but it is still difficult to solve the problem of financing difficulties, high risks and poor supervision in supply chain finance. However, with the development of blockchain technology Development, with its high credibility, strong traceability, non-tampering, decentralization and other characteristics. It can break through the current plight of supply chain finance development, and provide new ideas for financing, reducing financial risks, optimizing project screening, and standardizing project supervision.

Blockchain technology helps financial disintermediation. Realize point-to-point services, enable the supply and demand parties of resources to directly trade, reduce intermediate links, reduce agency costs caused by mismatched information, improve transaction security and efficiency, provide instant and high-quality services for both parties, optimize resource allocation, and further form a highly reliable industrial value chain. Achieving cross-level communication between multiple entities and enhancing mutual trust among enterprises will help establish multi-level corporate strategic cooperation, achieve efficient circulation of resources, activate capital circulation, and establish strategic relationships.

Blockchain technology helps reduce financing costs. After all kinds of small and medium-sized enterprises are on the chain, the information of large and medium-sized enterprises is digitized, which further makes the information value and promotes the mapping of entities and information. At the same time, through the information sharing and distributed ledgers of all entities on the chain, a highly credible credit system is formed, and smart contracts are used to strengthen the self-regulation and security of the blockchain, thereby making credit review, credit risk, and project management costs and difficulties. Further reduction, the risk premium will also be reduced, thereby further reducing the financing costs of supply chain finance and the real economy.

Blockchain technology helps supply chain finance to spread widely. With the on-chain of small and medium-sized enterprises, financial institutions that are traditionally concentrated in developed regions will be converted to a flatter organizational structure, and the cost of new outlets will be greatly reduced through blockchain technology. But it can expand service boundaries, guide the optimal allocation of resources, and break geographical restrictions and service barriers. This will reduce financial exclusion in underdeveloped regions and lower the threshold of supply chain finance, which will provide small and micro enterprises with more financial services and customize richer financial products. 


\section{ANALYSIS OF THE MECHANISM OF BLOCKCHAIN TECHNOLOGY DIRECTLY HITTING THE PAIN POINTS OF SUPPLY CHAIN FINANCE DEVELOPMENT}

\subsection{Blockchain technology breaks through "information islands" in supply chain finance}

In the traditional financial system, there is a corresponding industrial chain. There are upstream and downstream enterprises, central enterprises, and decentralized enterprises. However, due to information asymmetry, it is often difficult to communicate between enterprises with large hierarchical spans, and information is blocked, thus forming information barriers. .

With the widespread use of blockchain technology in supply chain finance, the gradual on-chain of multiple subjects, each topic can obtain its own corresponding blockchain node through the distributed ledger for information

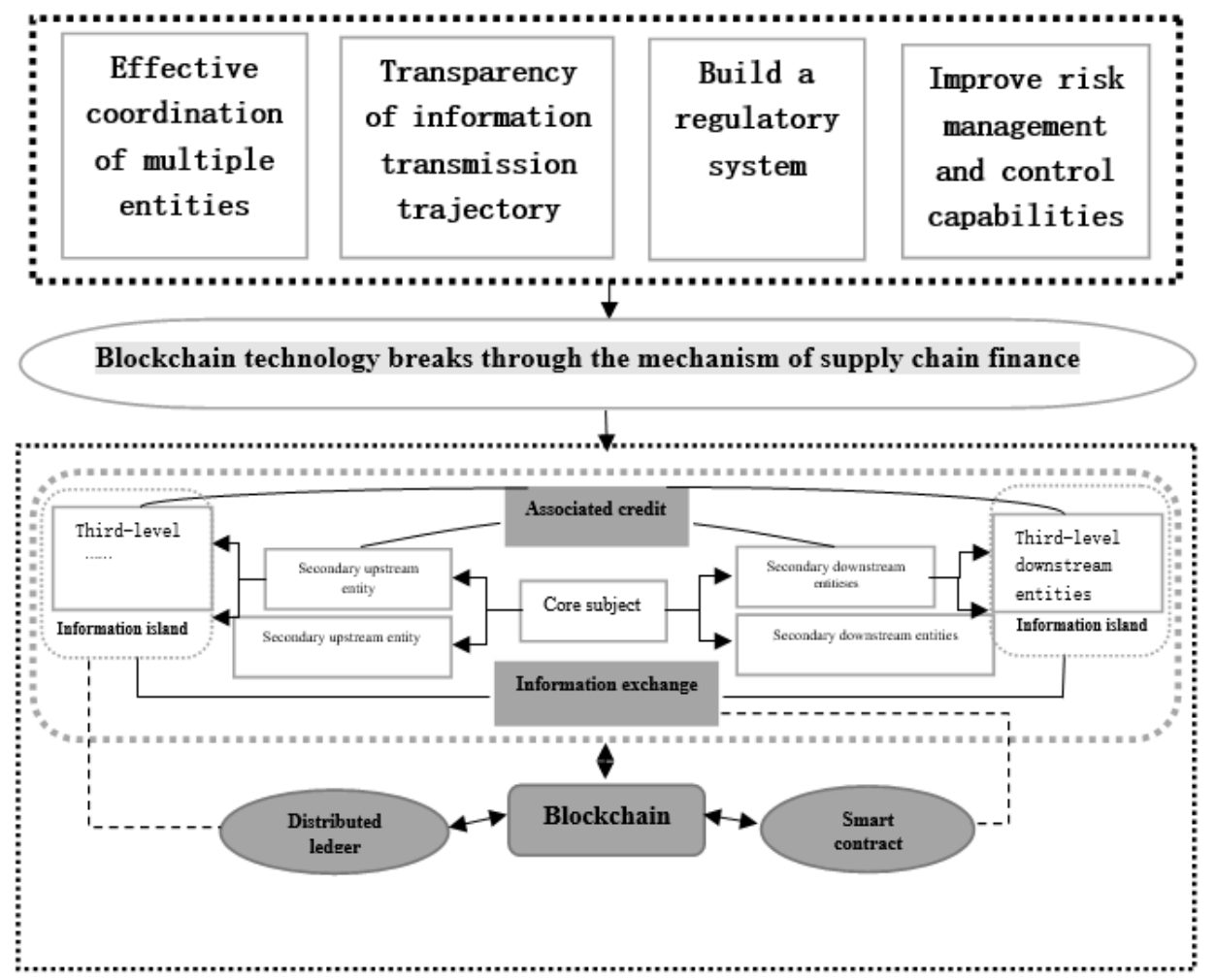

Figure 1 Blockchain technology optimizes supply chain finance

storage and recording. Since then, the information of the subject on the chain has been digitized, so that information can be exchanged efficiently and quickly in the blockchain system, thereby breaking the information barriers between enterprises, breaking information islands, and establishing connections and information exchanges between multiple subjects. It helps to establish mutual trust between enterprises, breaks the one-way transmission of information, forms an information network, links enterprises at all levels, promotes the formation of strategic alliances, promotes the flow of funds and resources, and strengthens the collaboration of various entities.

\subsection{Blockchain technology solves the financing difficulties and expensive financing of supply chain financial enterprises}

In traditional supply chain financial transactions, due to opaque transaction information and asymmetric information, serious principal-agent problems have arisen. The lenders represented by commercial banks cannot accurately understand the authenticity and unity of transactions between the entities, and cannot grasp the flow of funds and the status of the project in real time. As a result, the resulting financial risks have also risen, and this series of risks has further aggravated the problems of difficult and expensive financing for enterprises.

However, in the blockchain, due to the existence of network information interaction between entities at all levels, the information between the entities is updated in a timely manner, and the lower-level entities can obtain the credit line of the core entity through the split of bond shares during the transaction process, and use the 
associated credit to grant credit. The way to obtain a higher credit rating of the lender, thereby solving the problem of financing difficulties. Lenders can also use corporate information on the blockchain to directly establish credit associations with low-level companies to achieve peer-to-peer services, real-time supervision of contract progress and capital flow, reduce the transfer of intermediary agencies, improve transaction efficiency, establish strategic mutual trust, and reduce financing costs.

\subsection{Blockchain technology promotes the widespread deployment of supply chain finance}

As financial products and services are scarce resources in the economic market, there has long been a problem of uneven distribution of resources. Traditional large-scale financial service institutions are mainly concentrated in economically developed areas, while economically underdeveloped areas have relatively serious financial exclusion. It further deepens the problem of financing difficulties for enterprises in economically backward areas, and at the same time prevents the supply chain financial industry chain from spreading to undeveloped areas.

However, when financial companies are on the chain, they will greatly reduce the cost of setting up their institutions in underdeveloped regions. Both investment and financing parties use the blockchain information network model to exchange real-time information, optimize resource allocation, activate capital circulation, improve resource utilization efficiency, and optimize the rearrangement and combination of production factors. Alleviate financial exclusion in economically underdeveloped regions, and enable local economies to establish stronger strategic relationships, in order to realize the wide spread of supply chain finance, establish strong connections and strong communication between all levels.

\subsection{Blockchain technology strengthens supply chain financial market supervision}

As one of the most important links in the supply chain finance industry chain, all financiers represented by commercial banks attach great importance to risk supervision. However, in traditional transactions, due to the opacity of transaction information, there are certain difficulties and time differences in the publication and update of transaction details. A series of reasons have exacerbated information asymmetry and made supply chain finance increasingly fragile. Lack of supervisory bodies, difficult supervisory timing, backward supervisory methods, insufficient supervisory information, and non-timeliness have all made the supervision of the supply chain financial market deadlock, making all risk-sensitive financiers discouraged from investing.

However, the smart contracts and distributed ledgers in the blockchain can solve the above situation very well. As a necessary part of the blockchain, smart contracts are essentially the digitization and automation of traditional contracts. Through the immutable nature of distributed ledgers, effective supervision of both parties to the contract can only be started when the contract is signed. Only when the corresponding contract terms, legal obligations, and payment behavior are met, can the performance of the contract be triggered. At the same time, the information can be made transparent through the key and public key, so that the parties to the transaction can clarify the performance of the contract. Smart contracts combined with distributed ledgers can automatically supervise financial activities in the blockchain, which reduces the difficulty of supervision, improves supervision efficiency, and reduces the cost of transactions and supervision.

\section{STRATEGIC ANALYSIS OF THE DEVELOPMENT MODEL OF SUPPLY CHAIN FINANCE DRIVEN BY BLOCKCHAIN TECHNOLOGY}

Blockchain technology brings new vitality to supply chain finance, but challenges also follow. How to organically combine blockchain technology with supply chain finance and effectively drive the development of supply chain finance requires customized development based on the characteristics of supply chain finance, and its combination strategy is also particularly important.

\subsection{Encourage companies to take the initiative to go on the chain and expand the spread of blockchain technology}

First of all, a broad user base is required, and all parties in the supply chain need to understand and recognize the role of blockchain technology in driving supply chain finance, and actively store their own corporate behavior records in the distributed ledger. This behavior is essentially to digitize one's own information, thereby providing the possibility for the value of information. At the same time, it is necessary for all subjects to be able to actively share information, so that information can be transparent on the blockchain, thereby further achieving decentralization. It enables individuals at all levels to establish strategic mutual trust and promote the development of supply chain finance. With the gradual expansion of the user base, blockchain technology will gradually show a scale effect, so as to achieve information sharing between supply chains and achieve optimal allocation of resources. 


\subsection{Optimize the "blockchain+" supply finance model and strengthen the support of blockchain technology}

As an emerging technology spawned in the Internet era, there are more or less incompatibility problems when supply chain finance is combined. First of all, the information sharing pursued by the blockchain to achieve decentralization, while using asymmetric encryption technology to ensure corporate information security. However, supply chain finance has a large amount of sensitive information and commercial secrets in the transaction process, and it is not convenient for both parties to the transaction to disclose Therefore, there is a balance between this and information sharing. When blockchain technology is applied to supply chain finance, it is also necessary to upgrade its own encryption and anti-interception and anti-cracking technology levels to ensure user information security and safeguard users' economic interests. Secondly, because the distributed ledger technology cannot be tampered with, the data of each step of the user will be stored on the chain. With the expansion of users and the passage of time, a huge amount of data storage will be generated. Therefore, powerful data storage and transmission technology is also a key part of blockchain technology that needs to be improved.

\subsection{Develop a standardized mechanism and build a cross-blockchain strategic system}

The rapid and efficient information flow achieved within the blockchain project is not enough to meet the needs of current social and economic development. However, due to the differences in the internal programming languages of various blockchain projects and the differences in confidentiality agreements, the cross-chain interconnection between the blockchain and the blockchain has been caused. In order to form a scale effect, it is necessary to establish a standardization mechanism to generate synergy between various entities, improve the scalability and interaction between each blockchain, and build a complete supply chain financial system supported by blockchain technology.

\section{CONCLUSIONS}

In summary, this article starts from the core technology of blockchain and combines the demands of the current development of supply chain finance. With regard to blockchain technology, the current information barriers to supply chain finance are eliminated through distributed ledgers, speeding up the efficiency of information flow, and enhancing strategic mutual trust. Use technologies such as affiliated credit to solve contemporary corporate financing difficulties and expensive financing issues. And through smart contracts to effectively strengthen the supervision of enterprises, improve the credibility of transactions, reduce investment and financing risks and other aspects are discussed. But at present, the organic combination of blockchain technology and supply chain finance still has a long way to go. Blockchain technology needs to break through endogenous barriers, providing customized services for supply chain finance, and gain public recognition. Only by improving information security, formulating standardized regulations, and improving the regulatory system, can blockchain technology truly drive the development of supply chain finance.

\section{ACKNOWLEDGMENTS}

This article is supported by Graduate Innovation Foundation: Research on Integration and Innovation of "'Blockchain +' Ecological Agricultural Products Supply Chain_-Based on the case analysis of the 'Taste of Good Food' platform" Project Number: YJS2020005.

\section{REFERENCES}

[1] Masoud Kamalahmadi, Mahour Mellat Parast An assessment of supply chain disruption mitigation strategies [J] International Journal of Production Economics, 2017, $184 . \quad$ DOI: 10.1016/j.ijpe.2016.12.011

[2] Xu Zhenyu, Wu Jinping, Huo Yurong. Research hotspots and trends analysis of blockchain at home and abroad [J]. Library, 2019(04): 92-99. DOI:10.3969/j.issn.1002-1558.2019.04.015.

[3] Efanov D, Roschin P. The All-pervasiveness of the blockchain technology [J]. Procedia computer science, 2018, 123:116-121. DOI: 10.1016/j.procs.2018.01.019

[4] Ouyang Liwei, Wang Shuai, Yuan Yong, Ni Xiaochun, Wang Feiyue. Smart Contract: Architecture and Progress [J]. Acta Automatica Sinica, 2019, 45(03): 445-457. DOI: $10.16383 /$ j.aas.c180586.

[5] Yuan Yong, Ni Xiaochun, Zeng Shuai, Wang Feiyue. Development status and prospect of blockchain consensus algorithm [J]. Acta Automatica Sinica, 2018, 44(11):2011-2022. DOI: 10.16383/j.aas.2018.c180268.

[6] Zhao Xia, Wu Fangwei. Research on Revenue Sharing Contract of Agricultural Product Supply Chain Coordination under Random Output and Demand [J]. Chinese Management Science, 2009, 17(05):88-95.DOI:10.16381/j.cnki.issn1003-207x. 2009.05.008 